\title{
New method for route efficient energy calculations with mobile- sink for wireless sensor networks
}

\author{
Mohammad Khalaf Rahim Al-Juaifari, ${ }^{1,2,3}$, Jammel Mohammed Ali Mohammed Mona ${ }^{2}$, \\ Zainab Abd Abbas ${ }^{3}$ \\ ${ }^{1}$ Infromation Technology Research and Development Center (ITRDC), University of Kufa, Najaf, Iraq \\ ${ }^{2}$ Faculty of Medicine, University of Kufa, Najaf, Iraq \\ ${ }^{3}$ Faculty of Pharmancey University of Kufa, Najaf, Iraq
}

\begin{tabular}{l} 
Article Info \\
\hline Article history: \\
Received May 26, 2021 \\
Revised Oct 13, 2021 \\
Accepted Nov 23, 2021 \\
\hline
\end{tabular}

Keywords:

Base station

Energy consumption

Mobile sink

Node movement

Wireless sensor network

\begin{abstract}
Despite proposing a number of algorithms and protocols, especially those related to routing, for the purpose of reducing energy consumption in wireless sensor networks, which is one of the most important issues facing this type of network. In this research paper, energy consumption and cost are calculated taking into account energy consumption and the amount of data transferred to a thousand nodes through specific paths towards the mobile sink. The proposed model simulated by sending various amounts of data with specific path to know the energy consumption of each track and the network life time with 250, 500, and 1000 bits. Cost calculated using various weight for each track of these paths and the coefficient of movement time and path loss factor and others related to the transmission and receiving circuits. And finally, the results compared with a previous method it showed the efficiency of our method used and calculating 1000 nodes with various amount of bits to show the experimental results. Deep learning used to remember each and every path of each position or nearby to avoid calculation cost later.
\end{abstract}

This is an open access article under the CC BY-SA license.

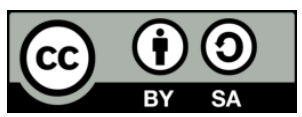

\section{Corresponding Author:}

Jammel Mohammed Ali Mohammed Mona

Faculty of Medicine, University of Kufa

Najaf, Iraq

Email: jammelm.mana@uokufa.edu.iq

\section{INTRODUCTION}

Wireless sensor networks (WSNs) used in many healths, military and agricultural applications and in monitoring land borders and others, especially in harsh environments such as desert, forests and rugged places or the use of some of these sensors to predict earthquakes and hurricanes. So, their presence in such environments makes it difficult to reach them for the purpose of providing them with the energy needed to survive. The network has all its live nodes, and the sensors also differ in the type of data they transmit and according to the purpose for which they were created were voice or image and other data.

Figure 1 shows movement steps towards the base station of sample network which follow changed the radius: $R$ is radius of networks, $0.95 R, 0.9 R, 0.8 R, 0.7 R, 0.6 R, 0.5 R, 0.4 R, 0.3 R, 0.2 R, 0.1 R$, and $0.05 R$ respectively moving trajectory and kept other parameters unchanged. Those means BS is located to each node from one to 1000 nodes each one located with its number towards BS which is before node movement, then move 0.95 rates from $\mathrm{R}$ which is started from new location $0.95^{*} 1$ for first nodes to $0.95 * 1000$ to last node, next move 0.9 rates from $\mathrm{R}$ which is started from new location $0.9^{*} 1$ for first nodes to $0.9^{*} 1000$ to last node, pass throw $0.8 \mathrm{R}, 0.7 \mathrm{R}, 0.6 \mathrm{R}, 0.5 \mathrm{R}, 0.4 \mathrm{R}, 0.3 \mathrm{R}, 0.2 \mathrm{R}, 0.1 \mathrm{R}$, and $0.05 \mathrm{R}$ with same scenario. 
Chain arrangement of send and receive packet from one node to its neighbor, packets collect finally in BS Cluster for choosing by calculated threshold value for each node. Energy is consumed per node in the operation of the node and also in the process of transferring data that increases its quantity in addition to being affected by the quality of data increase and decrease, in addition to other factors, which include the sink movement and constant of the path and the weight factor and the new distance, which will be addressed in the implementation part of this research. When studying the topic of energy saving, we find most of the research focused on the topic of energy conservation through the route and make improvements to it by choosing the best path to reach the BS and in various ways, varying between choosing the most energy path or choosing the closest neighbor node in the selection of paths up to the traditional methods of sleeping. When there is no energy efficiency in it. In this paper, 11 movements were used for every 1000 nodes in addition to the nodes in place before the movement, where the calculations for the energy consumed were performed during the operation of the node and after its movement for the movements that we referred to above to BS as shown in Figure 1, where data was compared quantities with 250, 500, and 1000 bytes of data in each of the aforementioned transfers, where the cost and the lowest cost were calculated and their relationship to the death of the contract in the specified time periods and locations and when you sink the sink a static move taking into account the weight factor of the contract and the new locations of the contract after each movement in addition to what was done. Calculate the remaining energy after each movement and calculate the critical energy for each. The use of 1000 nodes and a procedure described above is considered to be one of the first works of its kind in this quantity and with these calculations, which have arrived at the optimum values for use when creating WSNs of this size and those calculations for optimal selection of them when designing this type of network. Networks can contain one Mobile sink or multiple mobile sinks based on size of network, then load balance is required among all nodes; finally power distributed among all mobile node for each one mobile sink, also deep neuron network used to choose next path by train and testing the past calculations to reduce overall routing time for overall network.

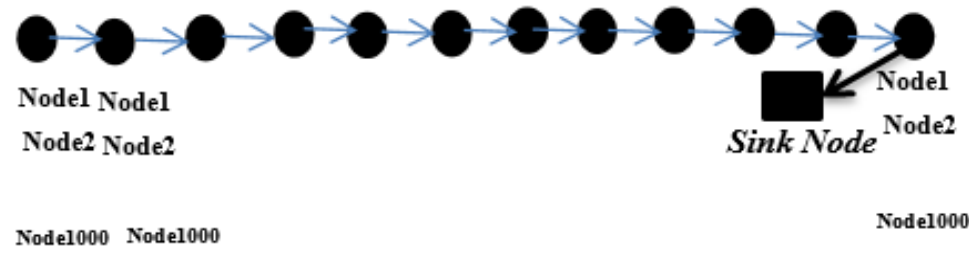

Figure 1. Random 1000 nodes topology

\section{PROPOSED SYSTEM MODEL AND PROBLEM STATEMENT}

\subsection{Background and literature review}

Many routing protocol used for energy efficient in WSNs such as in [1], adding the ability of balance the energy with enhancement in [2]. In [3] increase network lifetime for a multiple mobile sinks with distance-aware routing have been designed and utilized in mobile technology are ant colony optimization (ACO) or particle swarm optimization (PSO) algorithm; all protocols ant colony optimization for minimum cost coverage on 3-D grid (ACO-MCC3D) [4] designed for deployment optimization, ant colony optimization for travelling salesman problem (ACO-TSP) [5] for shortest path moving scheduling, in [2]-[4] FUCHAR are centralized topology control ACO Methods based, moreover single static sink used in [4]; while single mobile sink in [5] to increase network life time; while all protocols are practical swarn optimization PSO improvement [6] for balancing and energy efficient, energy-power management system (EPMS) [7] to increase network lifetime, and variable dimension particle swarm optimization (VD-PSO) [8] for path scheduling moving are PSO clustering with single mobile sink.

Shortest path used by many researchers to improve the power usage to decrease energy consumption by network. Shortest path calculations computed to avoid obstacles by a heuristic path planning algorithm presents with mobile sink in [9] using spanning graphs-based scheduling mechanism. In [10] author presents shortest path with a Hop based energy aware routing algorithm to up to $125 \%$ from existing algorithm at that time for WSNs also to decrease power consumption. Shortest path represents the most effective factor to save power with respecte to other factors.

Furthermore, some literature tries to optimize the performance of the network in aspects of energy consumption and lifetime by combining the particle swarm optimization algorithm [11], Apply efficient energy routing protocols or algorithms is the solution of energy shortage problem in WSNs [12], which is addressed efficiently energy and load balanced in [2], [13]. The selection of head cluster node [14] depends on value of the threshold to be head nodes which is mechanism of PEGASIS. Also in [5] collect data from 
node to node then send it via head cluster to base station. In [6] chain is reconstructed to head cluster to transmit data to BS in case of any node is dead. Wang et al. in [7] proposed to send and receive data to the head cluster using the threshold value if selected value is less than threshold value.

Clustering technique [8] efficiently optimize the power consumption in WSNs by dividing network sensors into specific clusters which depend on the type of those sensor, also in [9] clustering balance the energy based routing algorithm EEMSRA while balance of energy consumption data collection [10] which proposed TSP based best path routing for MS. In [14] prove the relation is increasing network lifetime by increasing number of mobile sink number. Variant MS. speed and direction: random MS. trajectory moving, static route moving as in [15], controllable moving as in [16], and [17] carry all types features using ETDC by balance energy during data collection then send it to mobile sink.

Efficient data gathering [18] using cluster-chain mobile agent routing (CCMAR) choosing optimal $\mathrm{CHs}$ by cluster head selection value (CHSV), optimal path schedule depend on both remaining energy level and signal strength. Routing decision making among residual energy, traffic, and path length of nodes in [19] applied Dempster Shafer evidence theory while unlike cluster routing protocols which has been proposed in [20] for balancing energy consumption for heterogeneous WSNs, while in [21] mutually path and route with various value of them.

Packet delivery Costs is reduced in [22] by collecting its neighbor sensors to find an optimal node. clustering protocols vs. flat routing protocol are: required less resources, scalable, better energy consumption and load balancing of sensor nodes, less packet overhead [23]. Some technologies such as Bluetooth, GPRS, WiMAX, and ZigBee [24] implemented based on applications and data rate, distance, frequency band, and power consumption. Routing protocol designed energy consumption sensing and transmitting data calculation the energy consumption at each node [25].

\section{RESEARCH METHOD}

The factors combined are influencing the choice of the best path to improve energy consumption in WSNs, as the calculation of one factor without the other does not represent a true criterion for the feasibility of using one technology without the other. There are many effective routing protocols used to improve energy efficiency performance and network life by introducing various algorithms in calculating residual energy. Directing multiple jumps to get from the node to the drain is preferred to reduce energy consumption to avoid the problem of hot spots.

\subsection{Network model}

Model contains number of nodes connected to mobile sink with clustering design in general. Proposed work implies nodes in sample network $\{\mathrm{n} 1, \mathrm{n} 2, \ldots\}$ arrangement as a circular shape distribution each group of nodes belong to one head cluster depend on the distance from each other carry initial power and sink velocity, nodes access mobile sink wireless any node within network with specific movement in Table 1. Standard values used to calculate the power in each and every position which is studied with value and unit for each parameter as listed in Table 1.

Table 1. Values of simulation parameters

\begin{tabular}{lcc}
\hline \multicolumn{1}{c}{ Parameter } & Value & Unit \\
\hline Number of nodes in network & $250,500,1000$ & \\
moving speed of sink & $\mathrm{Pi} / 5$ & meter/second \\
E elec: power consumption by Electrical components & $50 \mathrm{e}-9$ & $\mathrm{Jule}$ \\
L:Packet length & 500 & $\mathrm{bit}$ \\
$\alpha_{f s}$ Free space channel parameter & $10 \mathrm{e}-12$ & $\mathrm{Bit} / \mathrm{m}^{2}$ \\
$\alpha_{a m p}$ Multi-path channel parameter & $0.0013 \mathrm{e}-12$ & $\mathrm{Bit} / \mathrm{m}^{4}$ \\
Path loss parameter & 4 & \\
operational amplifier for Energy consumption & $5 \mathrm{e}-10$ & $\mathrm{Jule}$ \\
$\mathrm{E}_{\text {ini: Initial Energy }}$ & 0.05 & $\mathrm{Jule}$ \\
packet sending time interval & 5 & second \\
Adjusting parameter $(\alpha) \&(\beta)$ & $0.2,0.5$ & \\
\hline
\end{tabular}

\subsection{Energy model}

The Parameters $\alpha \& \beta$ is adjusting to 0.2 to increase network life time as proved in [12], moreover parameter 0.5 used later in to calculate cost experimental. Mobile Sink gather data describe method described in Figure 1. The result of the calculation in the division of 4 is shown by (1)-(10).

a) Transmission consumption signals generation \& its amplification which is according to transmission distance and reception consumption used as in [26] is calculated for 250,500, and 1000 bits data packet length respectively as shown: 
Transmit $(1, d)=\left\{\begin{array}{l}\text { L. } E_{\text {elec }}+\text { L. } \alpha_{\text {fs }} \cdot d^{n} \text { if } d_{i}<d_{i+1} \\ \text { L. E Elec }+ \text { L. } \alpha_{\text {amp }} \cdot d^{n} \text { if } d_{i} \geq d_{i+1}\end{array}\right\}$

where:

$E_{\text {elec: }}$ : consumption of electronic energy, $\alpha_{\mathrm{fs}}$ :free space model.

$\alpha_{\mathrm{mp}}$ : fading Multi-path model for long distance communication, $\mathrm{d}_{\mathrm{i}}$ : single hop communication distance.

b) Network life time calculation in (2):

$$
\text { Network lifetime }=\frac{\text { level of initial energy node } \mathrm{i}}{\text { node of enrgy consumption in each rounds }}
$$

c) New energy after movement to calculate required power consumption after movement for active node.

New energy after movement $=\mathrm{Ei}-\mathrm{Mpc}$.

Ei: initial energy, Mpc. Power consumption after movement.

d) Energy consumption calculated after movement to next portion towards MS as determined in (4).

$$
\text { Mpc. }=\text { L. } E_{\text {elec }}+\text { L. } \alpha_{a m p} \cdot d^{n} \text { if } d_{i} \geq d_{i+1}
$$

e) Active remaining energy node after communicate towards MS. After movement in (5):

R.E. I =New energy node- ST. *( MCE.)

R.E. is remaining energy, ST. sink time "in case of movement location".

f) Remaining energy of active node after movement towards MS.

$$
\text { R.E } \mathrm{i}=\sqrt{\frac{\sum_{\mathrm{i}=0}^{\mathrm{N}(\text { Ei-remaining eenrgy })^{2}}}{\mathrm{~N}}}
$$

i: nodes start with 1 end with 250 , d: distance from specific node to sink, $\mathrm{d}_{\mathrm{i}+1}$ : distance after movement of node as in Table 2 listed in section 4 . Other parameters assigned Table 1 in section 4.

g) Equation of cost with various factors.

$$
\text { Cost }=\min \left(\alpha .(\text { RE i }) 2+\beta \cdot \sum_{n=1}^{n}\left(\text { weight }_{i} * d \text { MS new }{ }_{i}\right)\right.
$$

dMSnew: distance to mobile sink with next movement. Where: weight $_{\mathrm{i}}$ shows step 8.

h) Calculate Weight of node time to still alive

$$
\text { weight }_{\mathrm{i}}=\frac{\text { Threshold node Remaining life time }}{\text { active node Remaining life time }}
$$

where $\mathrm{RL}_{\mathrm{i}}$ is remaining life time.

i) Node remaining life time described as in (9),

$$
\text { Rmeaning Life time }{ }_{i}=\frac{\text { Res }_{i}}{L\left(E_{\text {elec }}+\alpha * \text { distance toMSnew }_{i}^{n}\right)}
$$

j) Threshold energy is calculated using (10) to choose threshold to choose clusters (10):

$$
\text { Energy } \text { threshold }=\frac{\sum \text { EnegN } * \mathrm{E}_{\text {residental }}}{\mathrm{N}}
$$

\subsection{Procedure description}

Based on distance in [27] from node to mobile sink power is calculated required to communicate with neighbor node to send packet to mobile sink. Optimal communication distance can be calculated by enhancement of routing protocol of PEGASIS in [28] to increase network life time. There a lot of techniques to enhance Network lifetime such as in [12] one of important routing protocol has been presented to increase network life, it also discussed the life time of network with adjusting parameter, so that this paper focus on finding the cost using nearly same parameters and procedure listed in Table 1. 
1. Chain initialization.

2. Mobile sink calculate the location of each node by sending request and receiving it to construct cluster to initialize the head of this cluster.

3. By step 2 each particular cluster consists of number of nodes chooses by its locations, also energy remaining for each node in the network. Method is calculating distance from node to mobile sink in the network by the following: first allow for lowest distance to send data to mobile sink, second allow for fastest replay time for equal distance, third allow for highest distance node for sending packet to sink, next allow for minimum node power remaining to send packet to sink.

4. Calculate threshold value.

5. Go to step 2.

6. End step 2.

7. If Minimum node energy remaining equal to remaining energy.

8. End.

9. Mobile Sink now has optimal distance also nodes with less remaining energy.

10. Start next round if node dies.

\section{EXPERIMENTAL RESULT AND DISCUSSION}

Experimental results had been calculated based on Table 2 using selected position which covers almost the different palces manually. Such as authors in [29] use duty cycling \& data driven method to overcome problem of matching between sender and receiver, which shows values of locations after movement of nodes for sample network sensors as shown in Figure 1 to show movement of nodes to sink node method in proposed work used to increase network life time is to deplete node power consuming through different radius to center mobile sink. Later will usw these calculations to learn the network best path to use it in next routing in network wihich exactly or nearby the selected position.

Table 2. Nodes locations to mobile sink

\begin{tabular}{|c|c|c|c|c|c|c|c|c|c|c|c|}
\hline Radius (R) & Move 1 & Move 2 & Move 3 & Move 4 & Move 5 & Move 6 & Move 7 & Move 8 & Move 9 & Move 10 & Move 11 \\
\hline Node & $\mathrm{NR}=$ & $N R=$ & $\mathrm{NR}=$ & $\mathrm{NR}=$ & $\mathrm{NR}=$ & $\mathrm{NR}=$ & $\mathrm{NR}=$ & $\mathrm{NR}=$ & $\mathrm{NR}=$ & $\mathrm{NR}=$ & $\mathrm{NR}=$ \\
\hline $\begin{array}{l}\text { Movement } \\
\text { for Mobile } \\
\text { Sink }\end{array}$ & $0.05 \mathrm{R}$ & $0.1 \mathrm{R}$ & $0.2 \mathrm{R}$ & $0.3 \mathrm{R}$ & $0.4 \mathrm{R}$ & $0.5 \mathrm{R}$ & $0.6 \mathrm{R}$ & $0.7 \mathrm{R}$ & $0.8 \mathrm{R}$ & $0.9 \mathrm{R}$ & $0.95 \mathrm{R}$ \\
\hline
\end{tabular}

Simulation Figures 2 to 5 covers 1000 nodes range, packet=250,500, and 1000 bytes, same initial power, and other parameters showed in Tables 1 and 2 respectively. Figures 2(a)-(c) shows the cost with 250 bytes, 500 bytes and 1000 bytes respectively also Table 3 shows the minimum cost with changing factors of $\alpha \& \beta$ using parameters in Table 1 and 2 to clarify its relationship with energy consumption showed in Figure 4 with vary amount of data to be send along with location after movement for each node. Figures 2(a)-(c) shows changing locations from one position to another and its relationship with power consumption in each and every route from source to destination with various amounts of data. Large amount of data can be applied also but the changing becomes clearly defined with mentioned figures.

Figure 2(a) illustrates that the node 797 began to die and so on ascending until reaching node number 1000, as 796 is the last active node in this case with a card of 0.26472704 , then whenever the energy is increased to 0.0264728 the nodes live to the limit of node number 792 . In Figure 2(b) a illustrates that the node 670 began to die and so on ascending until reaching node number 1000, with energy is increased to be 0.529442598. In Figure 2(c) illustrates that the node 563 began to die and so on ascending until reaching node number 1000, as 796 is the last active node in this case with a card of 1.0588774 , then whenever the energy is to 0.26472704 "same 250 bytes" the node 709 die and from node 1 to node 708 nodes be alive.

Energy of nodes transmitting various amount of bytes 250,500, and 1000 respectively with radius distance based on Table 2 by calculating remaining energy and consumption power after movement. Simulation results shows that the Mobile Sink y is near lowest remaining energy to increase network life time, Figures 5(a)-(c) is calculating threshold values with various data transfer used by this work. Table 3 contains the cost with different life two adjusting parameters, impact of changing value is clearly.

Table 3. Minimum cost with variant value of $\alpha$ and $\beta$

\begin{tabular}{cccc}
\hline $\begin{array}{c}\text { Minimum cost with } R=0, \\
\alpha=\beta=0.2\end{array}$ & $\begin{array}{c}\text { Minimum } \operatorname{cost} R=0, \\
\alpha=\beta=0.5\end{array}$ & $\begin{array}{c}\text { Minimum cost } R=0.25 R, \alpha=\beta=0.2 \\
\text { with node trajectory }\end{array}$ & $\begin{array}{c}\text { Minimum cost } R=0.25 R, \alpha=\beta=0.5 \text { with } \\
\text { node trajectory }\end{array}$ \\
\hline 0.000307 & 0.003382 & 0.029286 & 0.073216246 \\
\hline
\end{tabular}




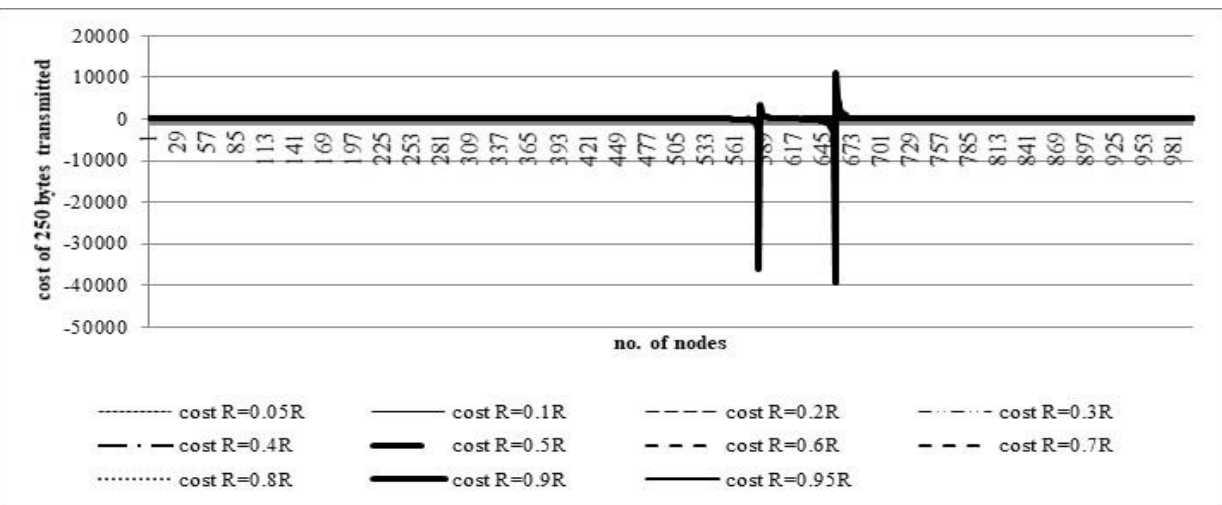

(a)

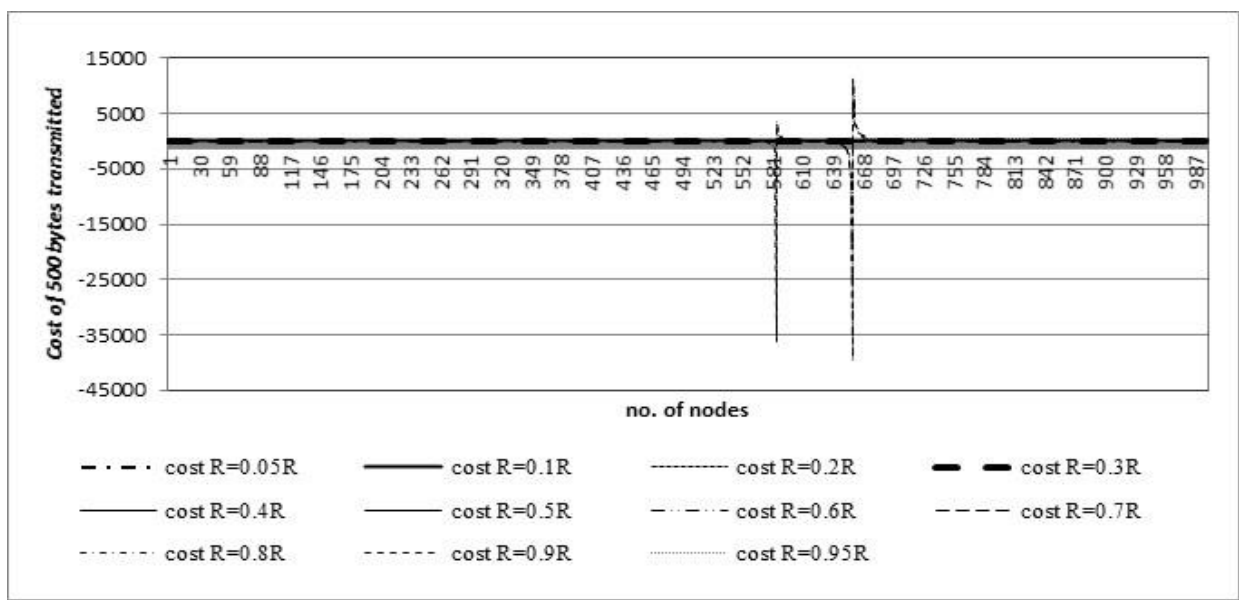

(b)

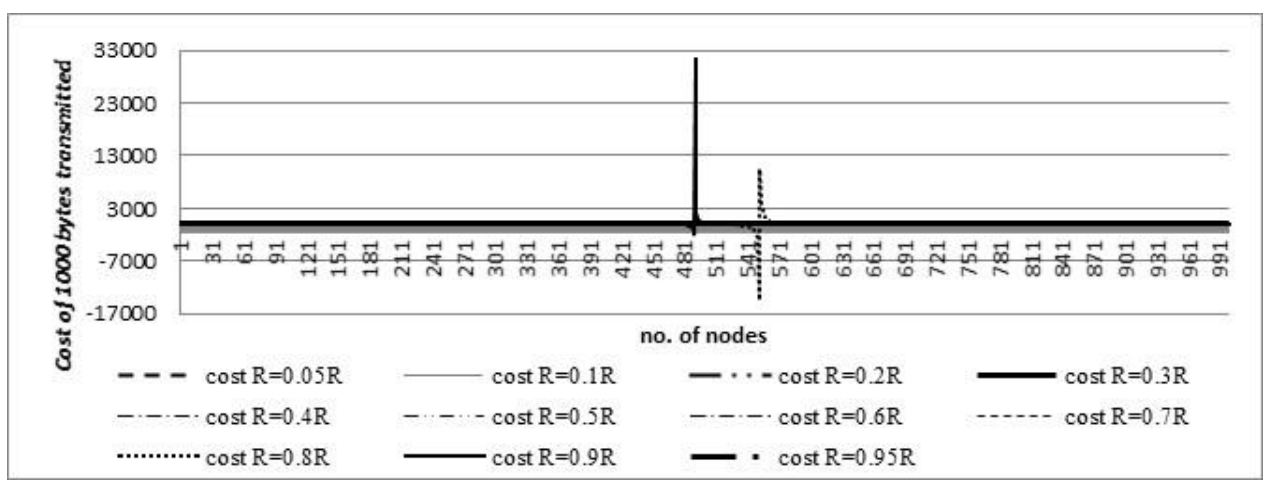

(c)

Figure 2. Cost of various data size to be transmitted; (a) 250 bytes transmitted, (b) 500 bytes transmitted, and (c) 1000 bytes transmitted

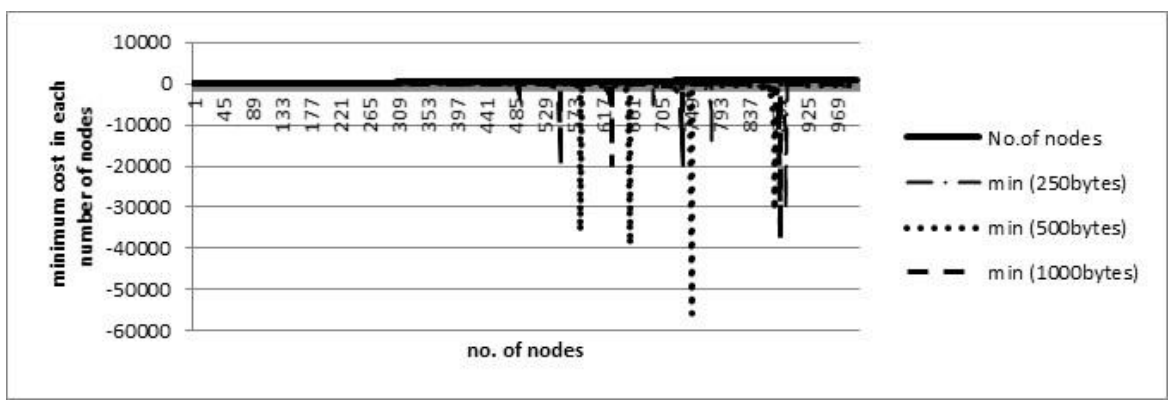

Figure 3. Minimum cost in each number of nodes 


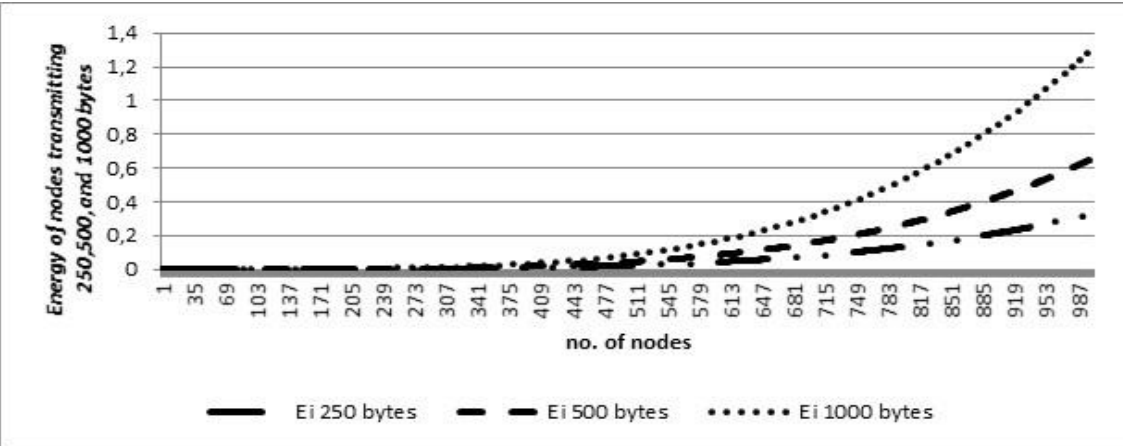

Figure 4. Energy of nodes transmitting 250, 500, and 1000 bytes

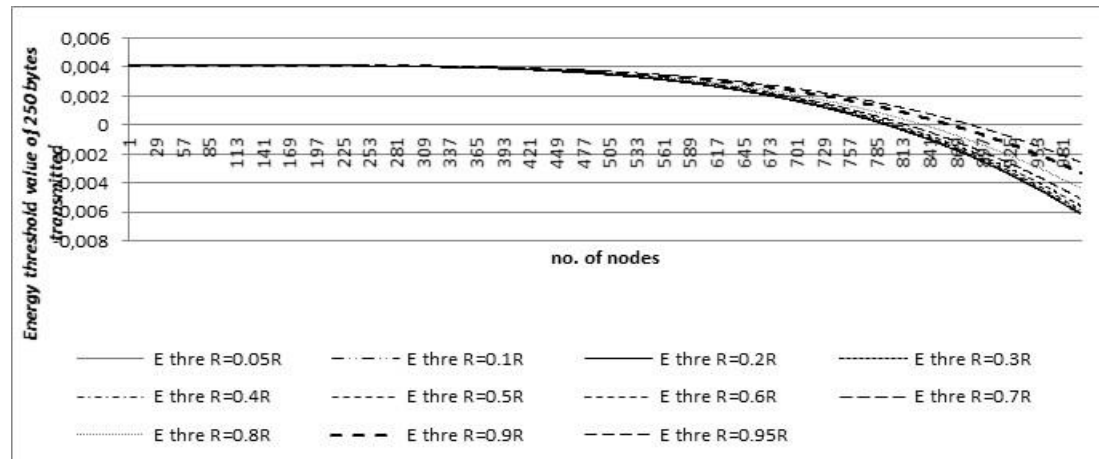

(a)

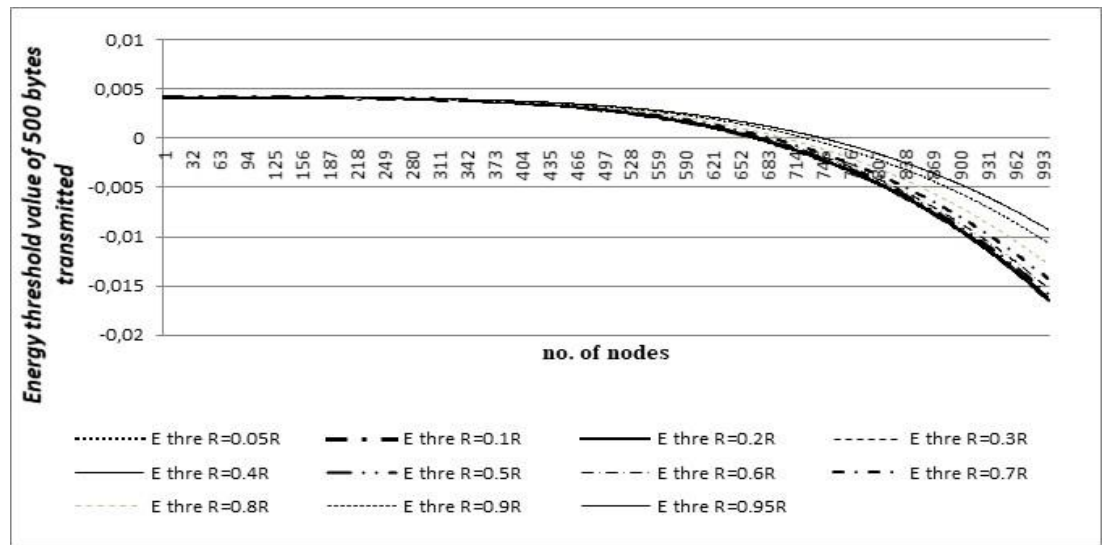

(b)

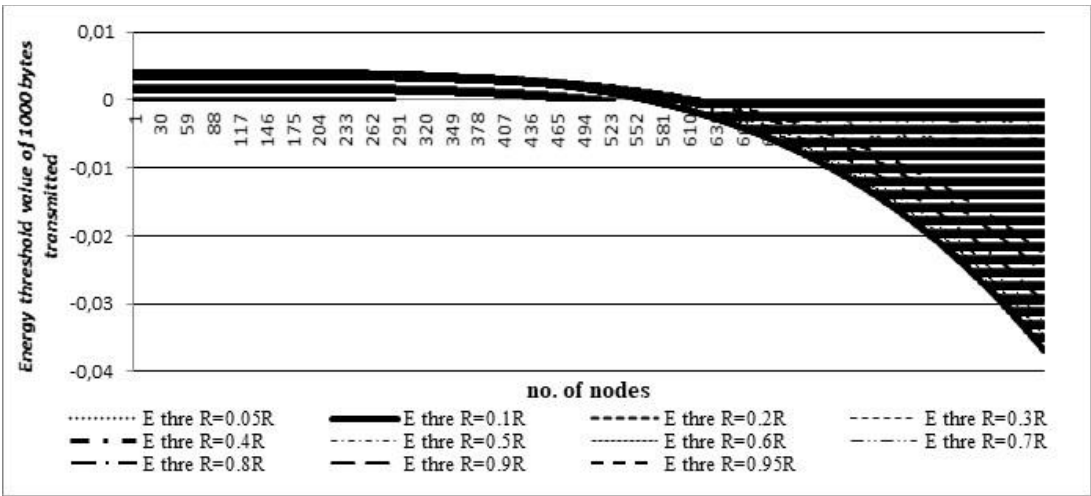

(c)

Figure 5. Energy threshold value of different data size to be transmitted: (a) 250 bytes transmitted, (b) 500 bytes transmitted, and (c) 1000 bytes transmitted 
Figures 5(a)-(c) shows that if the chosen value is less than the threshold value, it becomes the head of the cluster, otherwise it is a normal node which collects data then sends it to nearest neighbor which transmits data to the base station. Figure 6 shows network life time for energy consumption of node in each round calculated after applying proposed method in [12] with only $0,025 \mathrm{R}, 0.5 \mathrm{R}, 0.75 \mathrm{R}$ and $\mathrm{R}$ with 300 nodes and 500-bit packet length. Also, number of nodes becomes decrease network round life time which becomes stable after node 250 or nearby. Figure 6 uses only 500 bits to give clear indea and 500 is greater than 250 and less than 1000 bits to give only genral idea about the network life time. Figures 7(a)-(c) in this work extends of [12] with various packet length and 1000 number and nodes to calculate costs with more in Table 4 location as sample of experimental results.

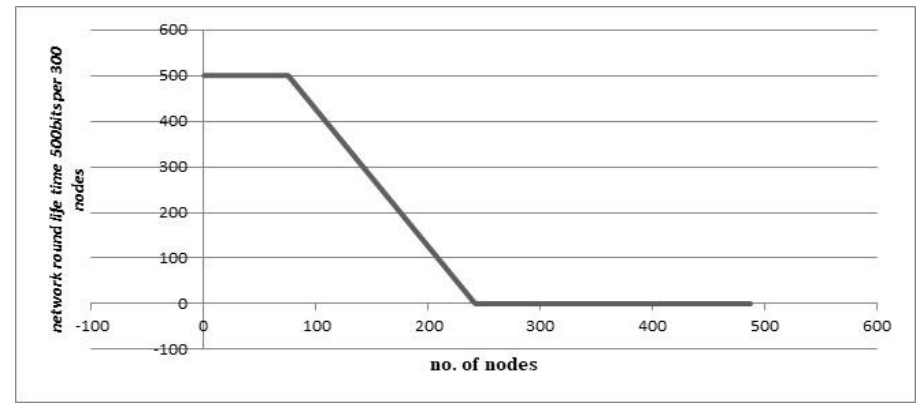

Figure 6. Network round life time 500bits per 300 nodes

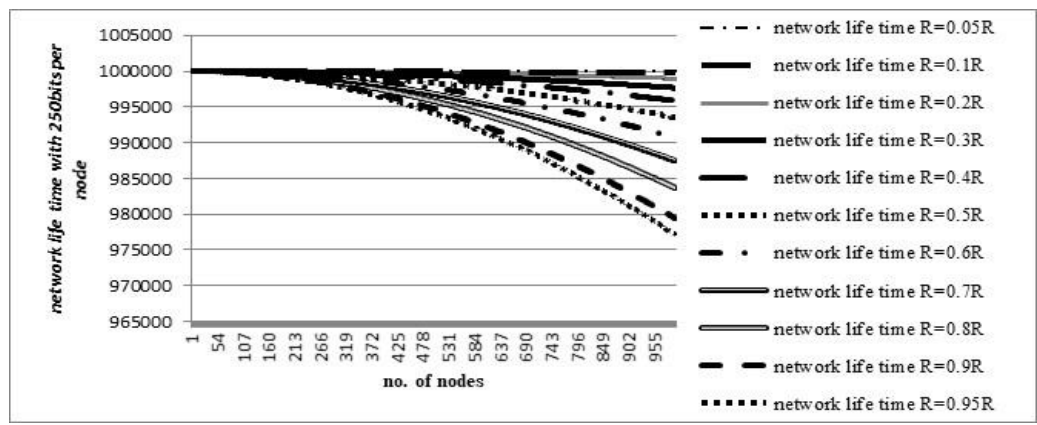

(a)

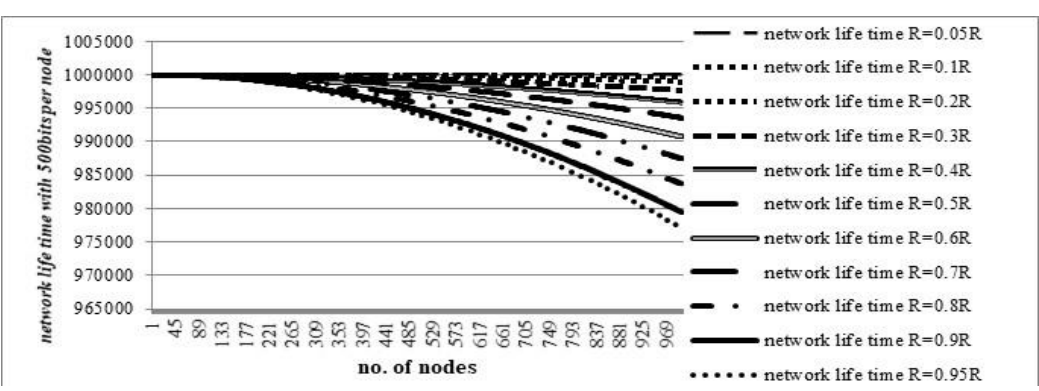

(b)

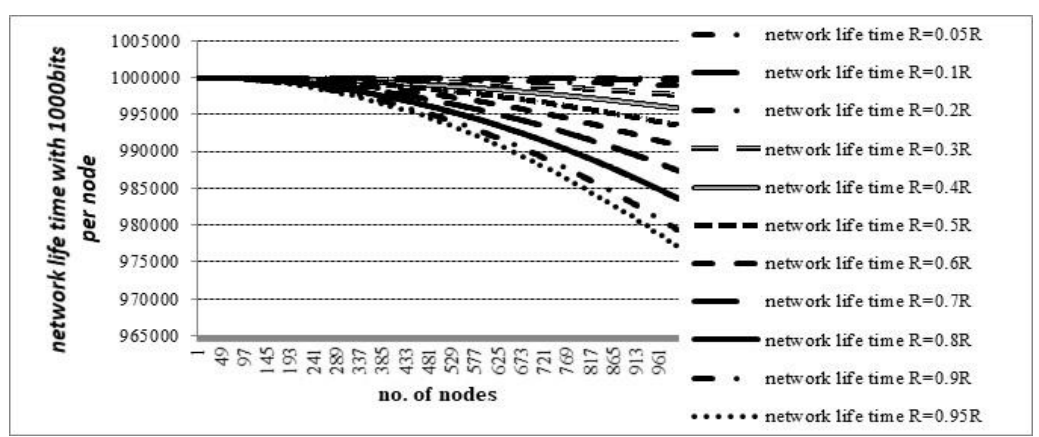

(c)

Figure 7. various data size of network life time with 1000 nodes: (a) 250 bits, (b) 500 bits, and (c) 1000bits 
Simulating results use link scheduling the packet by [30], [31] to improve network battery power by schedule the data which clearly showed the importance of node locations towards of mobile sink. Later methods and techniques as in [32]-[34] which used artificial intelligent to save power in distributed systems by using different machine learning techniques to save group by learn network paths for each and every node with this network. Improve network traffic performance and efficient path planning; also load balancing, traffic monitoring and firewall used for established applications [35], by collect the training data using Dijkstra's shortest static path algorithm to generate a collection of static paths then testing data. The accuracy of the training and testing in two-node constraint experiments and dataset size, beamwidth $=5$. Grid network Training0.999, Testing 0.999, and 250,500, and 1000 datasize for our sample network.

Table 4. Results for time consumed per node constraint in whole network

\begin{tabular}{cccc}
\hline Position & Throughput & Congest delay & Delay \\
\hline $\mathrm{R}$ & $1250.50 \mathrm{Mbps}$ & $85.23 \mathrm{~ms}$ & $0.27 .1 \mathrm{~ms}$ \\
$0.5 \mathrm{R}$ & $4350.2 \mathrm{Mb}$ & $22.15 \mathrm{~ms}$ & $8.18 \mathrm{~ms}$ \\
\hline
\end{tabular}

\section{CONCLUSION}

In this paper, the main contribution is that calculation of numerical cost to show the effect of changing value parameters on performance of whole network as well as factors in each step required using closely parameters in enhanced PEGASIS by presented and selecting specific position with various conditions to optimize network life time. Eventually, the calculations used for finding better path, for send and receive transmission data changing position to save power by managing transfer of packet; all of these factors have impact on network life time. Deep neurn network could be influenced to solve the problem of routing after learning the network path calculation to next to decided next route without calculations which speedup network routing, also deep learning can support sequence prediction to choose next hop with low cost.

\section{REFERENCES}

[1] M. T. Nguyen and N. Rahnavard, "Cluster-Based Energy-Efficient Data Collection in Wireless Sensor Networks Utilizing Compressive Sensing," MILCOM 2013 - 2013 IEEE Military Communications Conference, 2013, pp. 1708-1713, doi: 10.1109/MILCOM.2013.289.

[2] N. Zaman, T. J. Low, and T. Alghamdi, "Energy efficient routing protocol for wireless sensor network," 16th International Conference on Advanced Communication Technology, 2014, pp. 808-814, doi: 10.1109/ICACT.2014.6779072.

[3] Y. Gao, J. Wang, W. Wu, A. K. Sangaiah, and S-J. Lim, "A Hybrid Method for Mobile Agent Moving Trajectory Scheduling using ACO and PSO in WSNs,” Sensors, vol. 19, no. 3, 2019, doi: 10.3390/s19030575.

[4] T. Qasim et al., "An Ant Colony Optimization Based Approach for Minimum Cost Coverage on 3-D Grid in Wireless Sensor Networks," in IEEE Communications Letters, vol. 22, no. 6, pp. 1140-1143, June 2018, doi: 10.1109/LCOMM.2018.2819643.

[5] J. Wang, J. Cao, B. Li, S. Lee, and R. S. Sherratt, "Bio-inspired ant colony optimization based clustering algorithm with mobile sinks for applications in consumer home automation networks," in IEEE Transactions on Consumer Electronics, vol. 61, no. 4, pp. 438-444, November 2015, doi: 10.1109/TCE.2015.7389797.

[6] M. Azharuddin and P. K. Jana, "PSO-based approach for energy-efficient and energy-balanced routing and clustering in wireless sensor networks," Soft Comput., vol. 21, no. 6825-6839, 2016, doi: 10.1007/s00500-016-2234-7.

[7] J. Wang, Y. Cao, B. Li, H. Kim, and S. Lee, "Particle swarm optimization based clustering algorithm with mobile sink for WSNs,” Future Gener. Comput. Syst., vol. 76, pp. 452-457, 2016, doi: 10.1016/j.future.2016.08.004.

[8] W. Wang et al., "VD-PSO: An efficient mobile sink routing algorithm in wireless sensor networks," Peer-to-Peer Netw. Appl., vol. 10, pp. 537-546, 2016, doi: 10.1007/s12083-016-0504-x.

[9] G. Xie and F. Pan, "Cluster-Based Routing for the Mobile Sink in Wireless Sensor Networks With Obstacles," in IEEE Access, vol. 4, pp. 2019-2028, 2016, doi: 10.1109/ACCESS.2016.2558196.

[10] J. Wang, J. Cho, S. Lee, K-C. Chen, and Y-K. Lee, "Hop-based Energy aware routing algorithm for wireless sensor networks," IEICE Transactions on Communications, vol. E93.B, no. 2, pp. 305-316, 2010, doi:10.3390/s101009493.

[11] J. Wang, Y. Gao, W. Liu, A. K. Sangaiah, and H-J. Kim, “An Improved Routing Schema with Special Clustering using PSO Algorithm for Heterogeneous Wireless Sensor Network,” Sensors, vol. 19, no. 3, 2019, doi: 10.3390/s19030671.

[12] M. K. R. Al-juaifari, "Energy Cost Calculations for Enhanced Power Effiecient Gathering in Sensor Information Systems Algorithm with Mobile-Sink," Muthanna Journal of Pure Science, vol. 8, no. 1, 2021, doi: 10.52113/2/08.01.2021/14-21.

[13] Y. Ren, Y. Liu, S. Ji, A. K. Sangaiah, and J. Wang, "Incentive Mechanism of Data Storage Based on Blockchain for Wireless Sensor Networks," Mob. Inf. Syst., 2018, doi: 10.1155/2018/6874158.

[14] P. Zhong and F. Ruan, "An energy efficient multiple mobile sinks-based routing algorithm for wireless sensor networks," IOP Conf. Series: Materials Science and Engineering, vol. 323, doi: 2018, doi:10.1088/1757-899X/323/1/012029.

[15] A. Mehrabi and K. Kim, "Maximizing Data Collection Throughput on a Path in Energy Harvesting Sensor Networks Using a Mobile Sink," in IEEE Transactions on Mobile Computing, vol. 15, no. 3, pp. 690-704, 1 March 2016, doi: 10.1109/TMC.2015.2424430.

[16] H. Salarian, K. Chin, and F. Naghdy, "An Energy-Efficient Mobile-Sink Path Selection Strategy for Wireless Sensor Networks," in IEEE Transactions on Vehicular Technology, vol. 63, no. 5, pp. 2407-2419, Jun 2014, doi: 10.1109/TVT.2013.2291811.

[17] C. Sha, D. Song, R. Yang, H. Gao, and H. Huang, “A Type of Energy-Balanced Tree Based Data Collection Strategy for Sensor Network with Mobile Sink," in IEEE Access, vol. 7, pp. 85226-85240, 2019, doi: 10.1109/ACCESS.2019.2924919.

[18] S. Sasirekha and S. Swamynathan, "Cluster-chain mobile agent routing algorithm for efficient data aggregation in wireless sensor network," in Journal of Communications and Networks, vol. 19, no. 4, pp. 392-401, August 2017, doi: 10.1109/JCN.2017.000063. 
[19] L. Tang, Z. Lu, and B. Fan, "Energy Efficient and Reliable Routing Algorithm for Wireless Sensors Networks," Appl. Sci., vol. 10, no. 5, pp. 1885, 2020, doi: 10.3390/app10051885.

[20] Y. Liu, Q. Wu, T. Zhao, Y. Tie, F. Bai, and M. Jin, "An improved energy efficient routing protocol for wireless sensor networks," Sensors, vol. 19, no. 20, 2019, doi: 10.3390/s19204579.

[21] L. Hung, "The dynamic Routing for heterogeneous monitoring systems," 2017 International Conference on Applied System Innovation (ICASI), 2017, pp. 717-720, doi: 10.1109/ICASI.2017.7988529.

[22] A. Alromih and H. Kurdi, "An energy-efficient gossiping protocol for wireless sensor networks using Chebyshev distance," Procedia Comput. Sci., vol. 151, pp. 1066-1071, Jan. 2019, doi: 10.1016/j.procs.2019.04.151.

[23] P. C. S. Rao, P. K. Jana, and H. Banka, "A particle swarm optimization based energy efficient cluster head selection algorithm for wireless sensor networks," Wireless Networks, vol. 23, no. 7, pp. 2005-2020, 2017, doi: 10.1007/s11276-016-1270-7.

[24] D. M. Omar and A. M. Khedr, "ERPLBC-CS: energy efficient routing protocol for load balanced clustering in wireless sensor networks," Adhoc \& Sensor Wireless Networks, vol. 42, 2018.

[25] C. Del-Valle-Soto, C. Mex-Perera, J. A. Nolazco-Flores, R. Velázquez, and A. Rossa-Sierra, "Wireless Sensor Network Energy Model and Its Use in the Optimization of Routing Protocols," Energies, vol. 13, no. 3, pp. 728, 2020, doi: 10.3390/en13030728.

[26] J. Wang, Y. Gao, W. Liu, W. Wu, and S. Lim, "An Asynchronous Clustering and Mobile Data Gathering Schema based on Timer Mechanism in Wireless Sensor Networks," Comput. Mater. Contin., vol. 58, no. 3, pp. 711-725, 2019, doi: $10.32604 / \mathrm{cmc} .2019 .05450$.

[27] M. T. Nuruzzaman and H. Ferng, "A low energy consumption routing protocol for mobile sensor networks with a pathconstrained mobile sink," 2016 IEEE International Conference on Communications (ICC), 2016, pp. 1-6, doi: 10.1109/ICC.2016.7511316

[28] J. Wang, Y. Gao, X. Yin, F. Li, and H.-J. Kim, “An enhanced PEGASIS algorithm with mobile sink support for wireless sensor networks," Wireless Communications and Mobile Computing, vol. 2018, pp. 1-9, 2018, doi: 10.1155/2018/9472075.

[29] Z. Rezaei and S. Mobininejad, "Energy Saving in Wireless Sensor Networks," International Journal of Computer Science \& Engineering Survey (IJCSES), vol. 3, no. 1, pp. 23-37, 2012, doi: 10.5121/ijcses.2012.3103.

[30] H. Huang, H. Yin, G. Min, J. Zhang, Y. Wu, and X. Zhang, "Energy-Aware Dual-Path Geographic Routing to Bypass Routing Holes in Wireless Sensor Networks," in IEEE Transactions on Mobile Computing, vol. 17, no. 6, pp. 1339-1352, 1 June 2018, doi: 10.1109/TMC.2017.2771424.

[31] C. Wang, S. Guo, and Y. Yang, "An Optimization Framework for Mobile Data Collection in Energy-Harvesting Wireless Sensor Networks," in IEEE Transactions on Mobile Computing, vol. 15, no. 12, pp. 2969-2986, 1 Dec. 2016, doi: 10.1109/TMC.2016.2533390.

[32] Y. Lamalem, K. Housni, and S. Mbarki, "Enumeration of the minimal node cutsets based on necessary minimal paths," IAES International Journal of Artificial Intelligence (IJ-AI), vol. 9, no. 2, pp. 175-182, 2020, doi: 10.11591/ijai.v9i2.pp175-182.

[33] O. Garfi, H. Aloui, and N. Chaker, "Impacts of photovoltaic power source intermittence on a distribution network," International Journal of Electrical and Computer Engineering (IJECE), vol. 9, no. 6, pp. 5134-5142, 2019, doi: 10.11591/ijece.v9i6.pp5134-5142.

[34] M. D. Faraby, O. Penangsang, R. S. Wibowo, and A. Fitriati, "Coordinated planning in improving power quality considering the use of nonlinear load in radial distribution system," International Journal of Electrical and Computer Engineering (IJECE), vol. 11, no. 6, pp. 4610-4621, 2021, doi: 10.11591/ijece.v11i6.pp4610-4621.

[35] Y. Zuo, Y. Wu, G. Min, and L. Cui, "Learning-based network path planning for traffic engineering," Future Generation Computer Systems, vol. 92, pp. 59-67, 2019, doi: 10.1016/j.future.2018.09.043.

\section{BIOGRAPHIES OF AUTHORS}

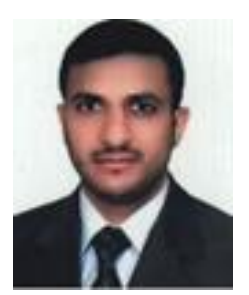

Mohammad Khalaf Rahim Al-juaifari (iD) 8d SC P Master of technology in computer science and engineering from JNTUH, Hyderabad 2012, India. He is a researcher and lecturer in University of Kufa, research interests are: wireless network power enhancement, virtualization, Data mining, He has published 7 research papers and currently submit three under assignment, also he is lecturer many topics in university of kufa such as compiler design, digital system design, microprocessor. He can be contacted at email: mohammad.aljuaifari@uokufa.edu.iq.

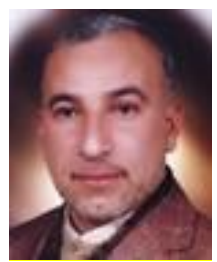

Jammel Mohammed Ali Mohammed Mona (D) 81 SC $P$ received the B.Sc. degrees in computer engineering from University of Technology, Baghdad, Iraq, in 1992, and M.Sc. degrees in software engineering from the University Technology, Malaysia, 2015. He is currently a Lecturer in the faculty of medicine, Najaf, university of Kufa, Najaf, Iraq. His research interests are in the area of software engineering, networking, cloud computing, iot, and wireless communications. He can be contacted at email: jammelm.mana@uokufa.edu.iq.

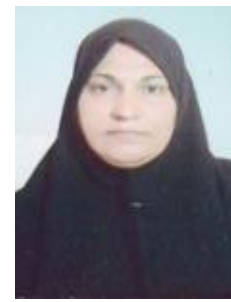

Zainab Abd Abbas (D) I8 SC P holds a certificate of B.SC from college of Sciences/AlMustansiriya University, she was the second on the Faculty of Sciences in (1994), Demonstrator in Kufa Medicine college/Al-Mustansiriya University in (1984), get Master's in Computer Science from Baghdad/National Center for Computers in (1989), Assistant lecturer in Kufa Medicine college/Al-Mustansiriya University in (1989), Assistant lecturer in Medicine college/AlMustansiriya University in (1990), publication of two papers at Al-Mustansiriya University, Assistant lecturer in Kufa University/College of Pharmacy (2005), Posted in Search Faculty of Medicine/University of Kufa in (2008), lecturer in College of Pharmacy/University of Kufa (2009), chief of computer center, organ in Iraqi computer society, participate in the Delphi program of the British Computer. She can be contacted at email: zainaba.abbas@uokufa.edu.iq. 